TM-1529

\title{
Antiproton Production and Energy Density Limitations in Targets for the Fermilab Pbar Source
}

I. L. Azhgirey and N. V. Mokhov Institute for High Energy Physics

Serpukhov, USSR

June 1988 


\title{
ANTIPROTON PRODUCTION AND ENERGY DENSITY LIMITATIONS \\ IN TARGETS FOR THE FERMILAB PBAR SOURCE
}

\author{
I.L.AZHGIREY, N.V.MOKHOV \\ Institute for High Energy Physics \\ Serpukhov, USSR
}

June 1988

The recent measurements of the antiproton yield [1] as well as the previous ones [2] differ from the predictions [3] which are the basis of the TEVATRON1 Design Report [4]. It was found in reference [1] that "at small acceptances, where the data depends essentially only on the forward pbar production cross section, the measured yield data indicates that these cross sections were over estimated by about a factor of 3 in the case of tungsten and about 2.3 in the case of copper". To clear up the situation and to understand what one can do to maximize the luminosity of the TEVATRON Collider this work has been done. Two sides of the antiproton production problem are considered:pbar production cross sections and targeting limitations.

Energy deposition density distributions in targets and particle yields are studied via Monte Carlo hadronic and electromagnetic cascade calculations. The calculations of reference [3] rely on the well known CASIM code [5]. In the present work we use two independent Monte Carlo programs : 
- MARS10, a fast inclusive program [6] which describes well microscopic and macroscopic data (see ref. $[7,8]$ ) in an arbitrary geometry for energies from $10 \mathrm{MeV}$ up to $30 \mathrm{TeV}$;

- FLUKA86, a program [9] with a full analog simulation of hadronic cascades on the basis of the multichain fragmentation model in the energy region from $50 \mathrm{MeV}$ up to $10 \mathrm{TeV}$.

Fig. 1 shows the antiproton momentum distributions at zero production angle from proton-tungsten collisions at $70 \mathrm{GeV}$ as calculated with the FLUKA86 code and as predicted in refs. $[3,10]$. Also shown is an experimental data fit from ref [2]. The $80 \mathrm{GeV}$ data of ref [10] have been divided by a factor of 1.2 to take into account an energy dependence of the cross section in this region. FLUKA86 results show no visible difference of the pbar yield in the $0-20 \mathrm{mrad}$ and $0-30 \mathrm{mrad}$ angular intervals for the $6-20 \mathrm{GeV} / \mathrm{c}$ momentum region and one can suppose that averaged over these intervals they are compatible with the zero angle data in the considered case. It is confirmed by direct calculation from formula [3] averaged over 0-25 mrad angular interval also shown in graph.

From Fig. 1 one can find the following numbers for antiproton yield averaged over the central region $(x=0)$ from 4 to $8 \mathrm{GeV} / \mathrm{c}: 0.05$ (data[2]), 0.085 (FLUKA86) and 0.10 (formula [3]). The corresponding ratio at 12 $\mathrm{Gev} / \mathrm{C}$ is $1: 2: 3.2$. It is important that there is no significant Adependence in the FLUKA86 predictions from $A 1$ to $W$, which is in good agreement with the conclusions of ref.[2].

Momentum distributions of antiprotons created in the $0-30 \mathrm{mrad}$ and $0-$ $60 \mathrm{mrad}$ angular bins when $120 \mathrm{GeV} / \mathrm{c}$ protons interact with tungsten nuclei are shown in fig.2. The figure compares curves taken from ref [3] with calculations of the FLUKA86 program.Again the Design Report curves lie higher than the FLUKA86 model predictions.

We have considered the targets and beam parameters which are the same as in the present Fermilab Pbar Source: $5 \mathrm{~cm}$ length tungsten and $7 \mathrm{~cm}$ length copper targets irradiated by $120 \mathrm{GeV} / \mathrm{c}$ protons with $0.1 \%$ momentum spread and with $0.7 \mathrm{mrad}$ FWHM divergence. The working point of the beam R.M.S. spot size is $0.378 \mathrm{~mm}$, but for energy density study we considered the $0.1-0.6 \mathrm{~mm}$ R.M.S. region.

To prepare a file for the consequent transport calculations in the AP2 beam line and Debuncher lattice,the produced negatives ( $p$, pi- and $\mathrm{K}-$ ) from the FLUKA86 program were collected in the various angular intervals with the momentum $\mathrm{PO} \neq 10 \%$ where $\mathrm{PO}=8.91058 \mathrm{GeV} / \mathrm{c}$. Table 1 shows the production rates of antiprotons for two targets. The difference in the yields of tungsten and copper targets is less than 20\%. The thickness of the targets in units of inelastic scattering lengths (FLUKA) is 0.47 for copper and 0.54 for tungsten. To express the yields in units of pbars per 
interacting proton one needs to multiply the $W / \mathrm{Cu}$ ratio of yields by factor $s=0.47 / 0.54=0.87$. After this the production rates on both targets will be practically the same (see last column of Table 1).

If one runs FLUKA86 at $70 \mathrm{GeV}$ for the conditions of ref [2] one get the following numbers for the antiproton production rate ratios: $W / C u=1.05$ and $W / A 1=1.11$, which coincides with the experiment[2]. The relative yield of other negatives on the Fermilab tungsten and copper targets are given in Table 2 for the $120 \mathrm{mrad}$ angular interval. The results for both materials coincide again.

The practical absence of A-dependence in p-bar yields gives one a chance to examine another target material besides tungsten, more suitable for higher proton beam intensity, to compensate the overestimation of the production cross section in the Design Report.

The smaller proton beam size results in a higher antiproton density in phase space. The maximum energy deposition density $\mathrm{E}_{\mathrm{m}}$ under interactions of the beam with targets, and the physical properties of target itself, determine the minimal spot size. Calculations of three-dimensional energy density distributions in targets intended for antiproton production were performed in ref.[4,11]. One obtained [4] the maximum energy density and tolerable beam intensity for $5 \mathrm{~cm}$ tungsten target. We have repeated these calculations with the modern tools FLUKA86 and MARS10 for tungsten and copper targets.

As was mentioned for the first time in ref.[11], when one calculates the energy density distribution for extremely small beam sizes and correspondingly extremely small radial bins special attention should be given to the details of very low energy particle transport which are normally deposited locally. The hadron threshold kinetic energy in CASIM and FLUKA86 is $\mathrm{E}_{\mathrm{th}}=50 \mathrm{MeV}$, in MARS10 was $\mathrm{E}_{\mathrm{th}}=10 \mathrm{MeV}$. FLUKA86 typically uses an empirical parametrization for electromagnetic showers energy deposition. During the simulation of associated electromagnetic showers in CASIM and MARS10, the electron and photon transport cut-off energy is 100 $\mathrm{keV}$, but the threshold energy at $e+e-$ production and bremstrahlung vertexes was $5 \mathrm{MeV}$.

Table 3 shows the fraction of the energy deposited locally in each cell. It illustrates the consequence of such approximations for the considered case of antiproton targets: up to $30 \%$ of energy in some particular cell could be incorrectly deposited locally. Moreover, in a run with poor statistics, fluctuations in small bins are completely determined by subthreshold particles. We have found the optimum values of the threshold energies: $2 \mathrm{MeV}$ for charge hadron transport and $200 \mathrm{keV}$ for EM-vertexes. We have put them in the present version of MARS10. The result: in all cases the input from locally deposited particles with 
energies below the new thresholds is less than 2\%.All MARS10 results in this paper have been obtained with this version.

The energies deposited in $5 \mathrm{~mm}$ radius and $7 \mathrm{~cm}$ length tungsten and copper targets via various chanels calculated with MARS10 and FLUKA86 are given in Table 4. The results of the two programs agree well. The main input comes from electromagnetic showers for both targets: for tungsten number is $85 \%$. Relations between the energy density components in longitudinal distributions differ fairly seriously for the two materials (figs. 3,4 ).

The radial distributions of energy density at the upstream and downstream ends of the tungsten and copper targets are shown in fig.5 and 6. Despite the difference in the threshold energies in MARS10 and FLUKA86, results of these two programs agree very well. For the copper target the results of a special MARS10 run with increased thresholds as well as energy deposition of primary protons at the beginning of the target are given.CASIM data from the Design Report [4], coinciding with others for the first longitudinal bin, lie up to 3 times below MARS10 and FLUKA86 results in the cascade maximum region(fig.5).A.Van Ginneken re-ran expresselly the present version of CASIM and found a much different answer. These new data are shown in Fig.5. Now all three programs are in reasonable agreement.

Usage of the Design Report data resulted in underestimation of the maximum energy density $\mathrm{E}_{\mathrm{m}}$ and correspondingly in an overestimation an admissible beam intensity. Fig.7 shows $E_{m}$ as a function of beam R.M.S. spot size. The MARS10 results are fitted well by

$$
\mathrm{E}_{\mathrm{m}}=\mathrm{C} \times \sigma^{-\mathrm{n}}, \text { for } \sigma=\sigma \mathrm{x}=\sigma \mathrm{y} \text { in } \mathrm{mm},
$$

where for tungsten $\mathrm{C}=0.6 \mathrm{GeV} / \mathrm{g}, \mathrm{n}=1.0$ and for copper $\mathrm{C}=0.135 \mathrm{GeV} / \mathrm{g}, \mathrm{n}$ $=1.35$. With the exception of one point the old data $[3,4]$ lie below, but the present CASIM result agrees very well with the MARS10.

There are two possible limits for target behaviour under irradiadiation by high intensity beam: melting point (which is $3410^{\circ} \mathrm{C}$ for tungsten and $1083{ }^{\circ} \mathrm{C}$ for copper) and shock waves limit. Experts have agreed[12] that for metal targets the last limitation is a priority and that the limit for $\mathrm{E}_{\mathrm{m}}$ is 200 Joules/g.Beyond this point shock waves can result in the destruction of the target. The CERN experience confirms this conclusion. The temperature which corresponds to this limit is $1500{ }^{\circ} \mathrm{C}$ for tungsten and $760{ }^{\circ} \mathrm{C}$ for copper. Now if one knows the maximum energy density $\mathrm{E}_{\mathrm{m}}$ in the target per one proton it is easy to 
find the tolerable number of beam protons $\mathrm{Np}$. For adiabatic case

$$
\mathrm{E}_{\mathrm{m}} \times \mathrm{N}_{\mathrm{p}} \times 1.6 \times 10^{-10}=200 \mathrm{~J} / \mathrm{g},
$$

where $\mathrm{E}_{\mathrm{m}}$ is in $\mathrm{GeV} / \mathrm{g}$.

Fig. 8 shows $N_{p}$ versus beam spot size for the tungsten and copper targets as calculated with MARS10 and fitted by

$$
\mathrm{N}_{\mathrm{p}}=\begin{aligned}
& 2.1 \times 10^{12} \times \sigma \text { for tungsten, } \\
& 9.3 \times 10^{12} \times \sigma^{1.35} \text { for copper }
\end{aligned}
$$

The Design Report data for tungsten more than twice overestimate our data for $\sigma>0.2 \mathrm{~mm}$, but the present CASIM's point is in excellent agreement again.

So, the copper target should be recomended for the PBAR Source. Tolerable beam intensity on such target is three times higher than for tungsten and antiproton yield is practically the same.

The authors wish to thank Gerry Dugan and Andy Van Ginneken for stimulating discussions and their very helpful comments on this paper. 


\section{REFERENCES}

1. G.Dugan. Pbar Yield Measurements vs. Debuncher Acceptance and Comparisons with Predictions. PBAR Note 475, Fermilab, 1988.

2. L.M.Barkov et al. Zeitschrift fur Physik, vol.C14,1982,pp.1-6.

3. C.Hojvat, A.Van Ginneken. Nucl.Instr. and Methods, vo1.206, 1983, pp.67-83.

4. Design Report TEVATRON1 Project, Fermilab, September 1984.

5. A.Van Ginneken. Fermilab FN-272,1975.

6. N.V.Mokhov. Preprint IHEP 82-168,Serpukhov, 1982. N.V.Mokhov, J.D.Cossairt. Nucl.Instr.and Methods, vol.A244, 1986,pp.349-355.

7. N.V.Mokhov, S.I.Striganov, A.V.Uzunian. Preprint IHEP 87-59, Serpukhov, 1987.

8. N.V.Mokhov. Sov.J.Part.Nuc1 , , vol.18(5), Sept.-0ct.1987, p.408

9. P.Aarnio, A.Fasso, H.Mohring, J.Ranft, G.Stevenson. CERN TIS-RP/168, 1986.

10. H.Mohring, J.Ranft. Nucl.instr.and Methods, vol.201, 1982 , pp.323-327.

11. N.V.Mokhov, A.Van Ginneken. Fermilab TM-977, 1980.

12. Proc. of High Intensity Targeting Workshop, Fermilab, 1980. 
Table 1. Antiproton production yields from the target.

\begin{tabular}{ccccc}
$\begin{array}{c}\text { Collection angle, } \\
\text { mrad }\end{array}$ & $\begin{array}{c}\text { Antiprotons, } \\
W\end{array}$ & $\begin{array}{c}\text { 1.E-3 } \\
\text { Cu proton }\end{array}$ & W/Cu & $s * W / C u$ \\
\hline$<120$ & 1.12 & .956 & 1.17 & 1.02 \\
$<90$ & 1.02 & .877 & 1.16 & 1.01 \\
$<60$ & 0.81 & .673 & 1.20 & 1.05 \\
$<30$ & 0.30 & .253 & 1.19 & 1.03
\end{tabular}

*) $s=0.47 / 0.54=0.87$

Table 2. Relative yield of negatives.

\begin{tabular}{|c|c|c|}
\hline Target & Tungsten & Copper \\
\hline $\begin{array}{l}\mathrm{pbar} / \mathrm{pi}- \\
\text { pbar/K- }\end{array}$ & $\begin{array}{l}.025 \\
.30\end{array}$ & $\begin{array}{l}.025 \\
.28\end{array}$ \\
\hline
\end{tabular}


Table 3. Relative energy deposition at $\mathrm{E}<\mathrm{E}_{\text {th }}$.

\begin{tabular}{|c|c|c|c|c|c|c|c|}
\hline \multirow[b]{3}{*}{$E_{t h}, \mathrm{MEV}$} & \multicolumn{2}{|c|}{$:$} & \multicolumn{2}{|c|}{$0<\mathrm{r}<0.01 \mathrm{~cm}$} & \multicolumn{3}{|c|}{$0.01<r<0.5 \mathrm{~cm}$} \\
\hline & : & $\mathbf{P}$ & $\pi \pm$ & EM-vertex & $\mathbf{P}$ & $\pi \pm$ & EM-vertex \\
\hline & $\vdots$ & 50 & 50 & 5. & 50 & 50 & 5. \\
\hline $\mathrm{Z}, \mathrm{cm}$ & $:$ & & & TUNGS & ISTEN & & \\
\hline $\begin{array}{l}0-1 \\
1-2 \\
2-3 \\
3-4 \\
4-5 \\
5-6 \\
6-7\end{array}$ & $\begin{array}{l}: \\
\vdots \\
\vdots \\
\vdots \\
\vdots \\
\vdots\end{array}$ & $\begin{array}{l}.2804 \\
.1825 \\
.0707 \\
.0949 \\
.0513 \\
.1179 \\
.0591\end{array}$ & $\begin{array}{c}0 . \\
0 . \\
.0100 \\
0 . \\
.0172 \\
.0154 \\
0 .\end{array}$ & $\begin{array}{l}0 . \\
0 . \\
0 . \\
.3667 \\
0 . \\
0 . \\
0 .\end{array}$ & $\begin{array}{ll}: & .1396 \\
: & .0486 \\
: & .0316 \\
\vdots & .0331 \\
\vdots & .0306 \\
: & .0283 \\
: & .0332\end{array}$ & $\begin{array}{l}.0100 \\
.0137 \\
.0071 \\
.0070 \\
.0095 \\
.0055 \\
.0219\end{array}$ & $\begin{array}{l}.0447 \\
.1723 \\
.2367 \\
.2180 \\
.2276 \\
.2787 \\
.2257\end{array}$ \\
\hline $\mathrm{Z}, \mathrm{cm}$ & : & & & & JPPER & & \\
\hline $\begin{array}{l}0-1 \\
1-2 \\
2-3 \\
3-4 \\
4-5 \\
5-6 \\
6-7\end{array}$ & $\begin{array}{l}: \\
\vdots \\
\vdots \\
\vdots \\
\vdots \\
:\end{array}$ & $\begin{array}{l}.1930 \\
.2050 \\
.1204 \\
.1572 \\
.1002 \\
.0743 \\
.0874\end{array}$ & $\begin{array}{c}0 . \\
0 . \\
0 . \\
0 . \\
.0217 \\
.0090 \\
.0152\end{array}$ & $\begin{array}{l}0 . \\
0 . \\
0 . \\
0 . \\
0 . \\
0 . \\
0 .\end{array}$ & 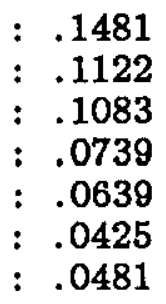 & $\begin{array}{l}.0091 \\
.1082 \\
.0305 \\
.0200 \\
.0102 \\
.0045 \\
.0253\end{array}$ & $\begin{array}{c}0 . \\
.0001 \\
.0365 \\
.0315 \\
.0219 \\
.0582 \\
.0754\end{array}$ \\
\hline
\end{tabular}

Table 4. Energy deposition components (GeV) for $120 \mathrm{GeV}$ proton.

\begin{tabular}{|c|c|c|c|c|}
\hline \multirow{2}{*}{ Component } & \multicolumn{2}{|c|}{ Tungsten } & \multicolumn{2}{|c|}{ Copper } \\
\hline & : MARS10 & FLUKA86 & : MARS10 & FLUKA86 \\
\hline Ionization & $: \quad .83$ & 1.05 & .33 & .39 \\
\hline EMS & $: \quad 9.30$ & 8.35 & .90 & .73 \\
\hline Nucl.excitation & .55 & .94 & .11 & .25 \\
\hline$E<$ Eth & $: \quad-$ & .22 & - & .07 \\
\hline Leakage & $: 109.0$ & 109.5 & 118. & 117.8 \\
\hline
\end{tabular}

*) The nuclear excitation component includes total excitation energy in FLUKA86 and only energy deposition from particles after de-excitation stage in MARS10. 


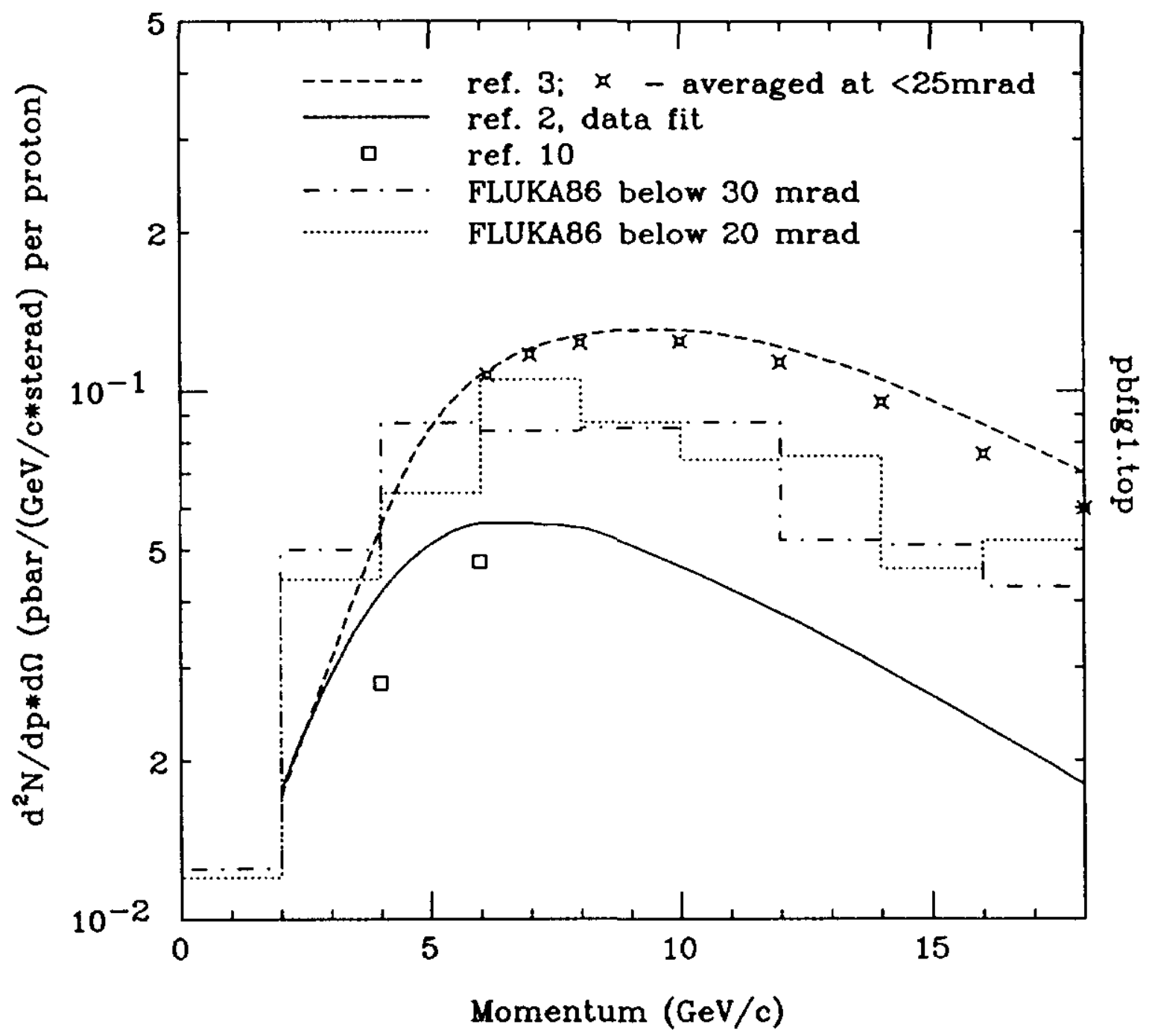

Fig.1. The pbar momentum distribution at zero engle for $70 \mathrm{GeV} / \mathrm{c}$ proton-tungsten interaction as predicted by various authors. 


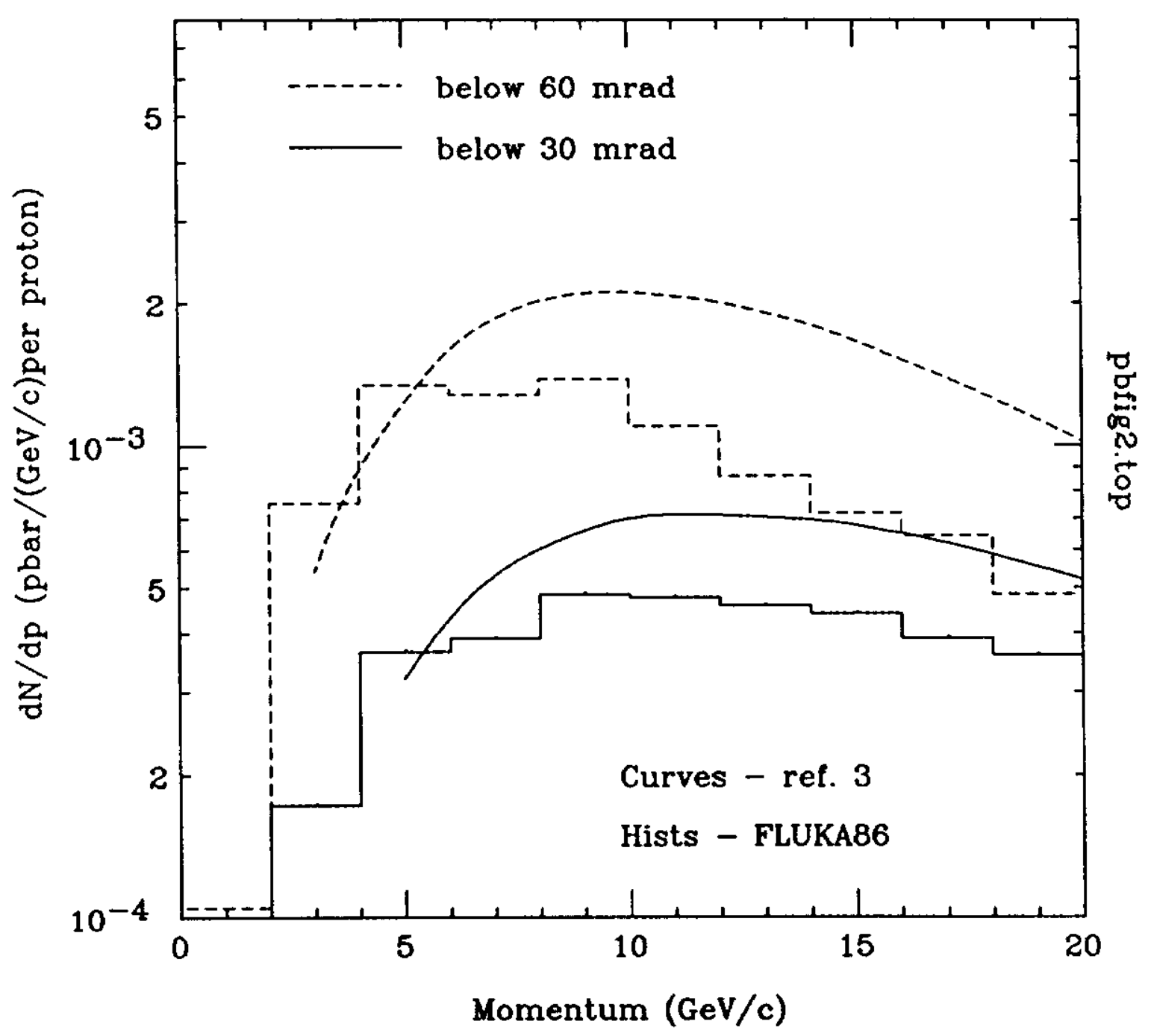

Fig.2. Integrated over two angular bins entiproton momontum distribution for $120 \mathrm{GoV} / \mathrm{c} \mathrm{pW}$ intersetions from rof.[3] and calculated in the present paper. 


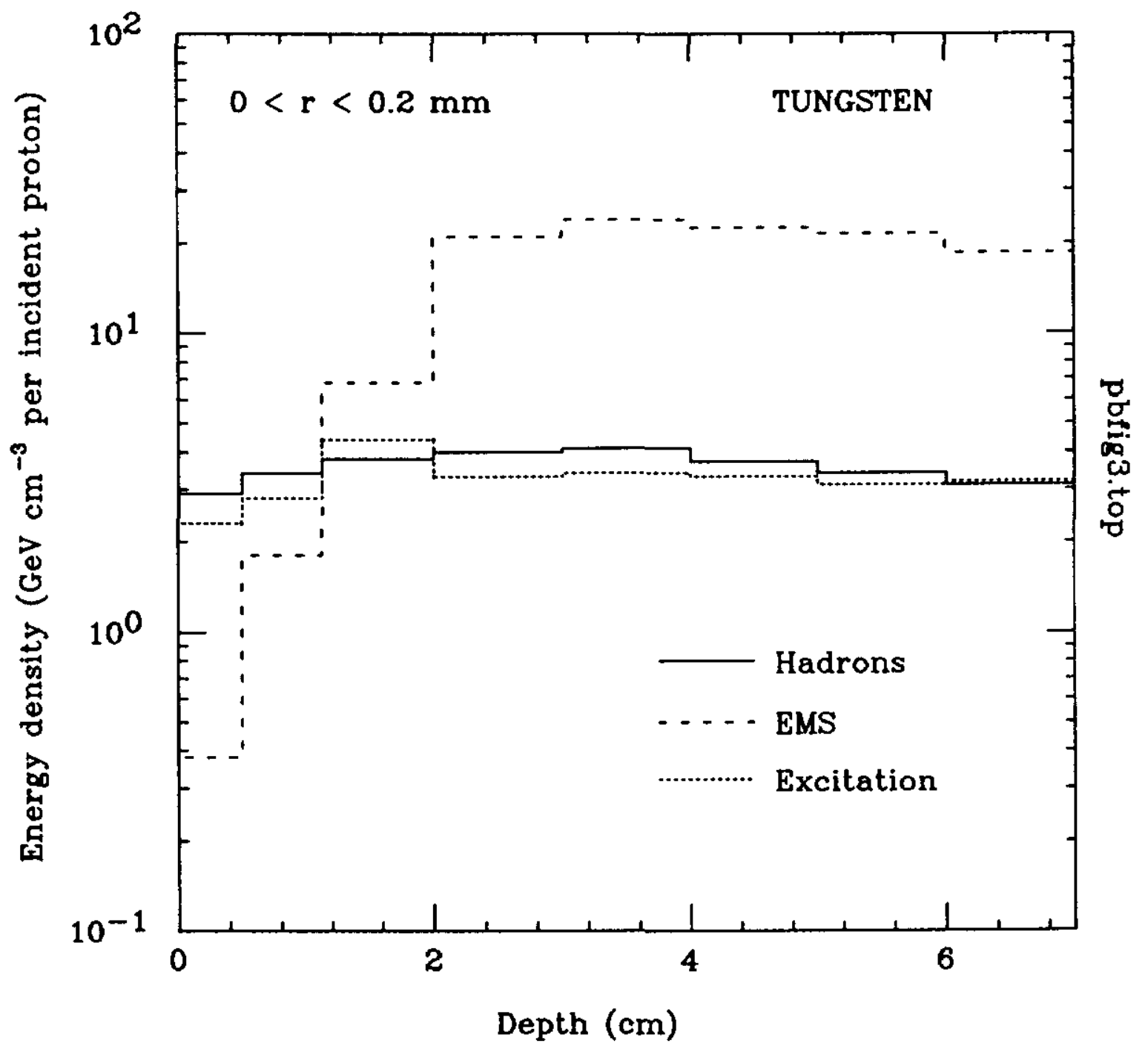

Fig.3. Longitudinal distibutions of energy density components around the beam axis in the Fermilab Pbar Source tungeten terget at $120 \mathrm{Ger} / \mathrm{c}$ as colculated with MARS10. 


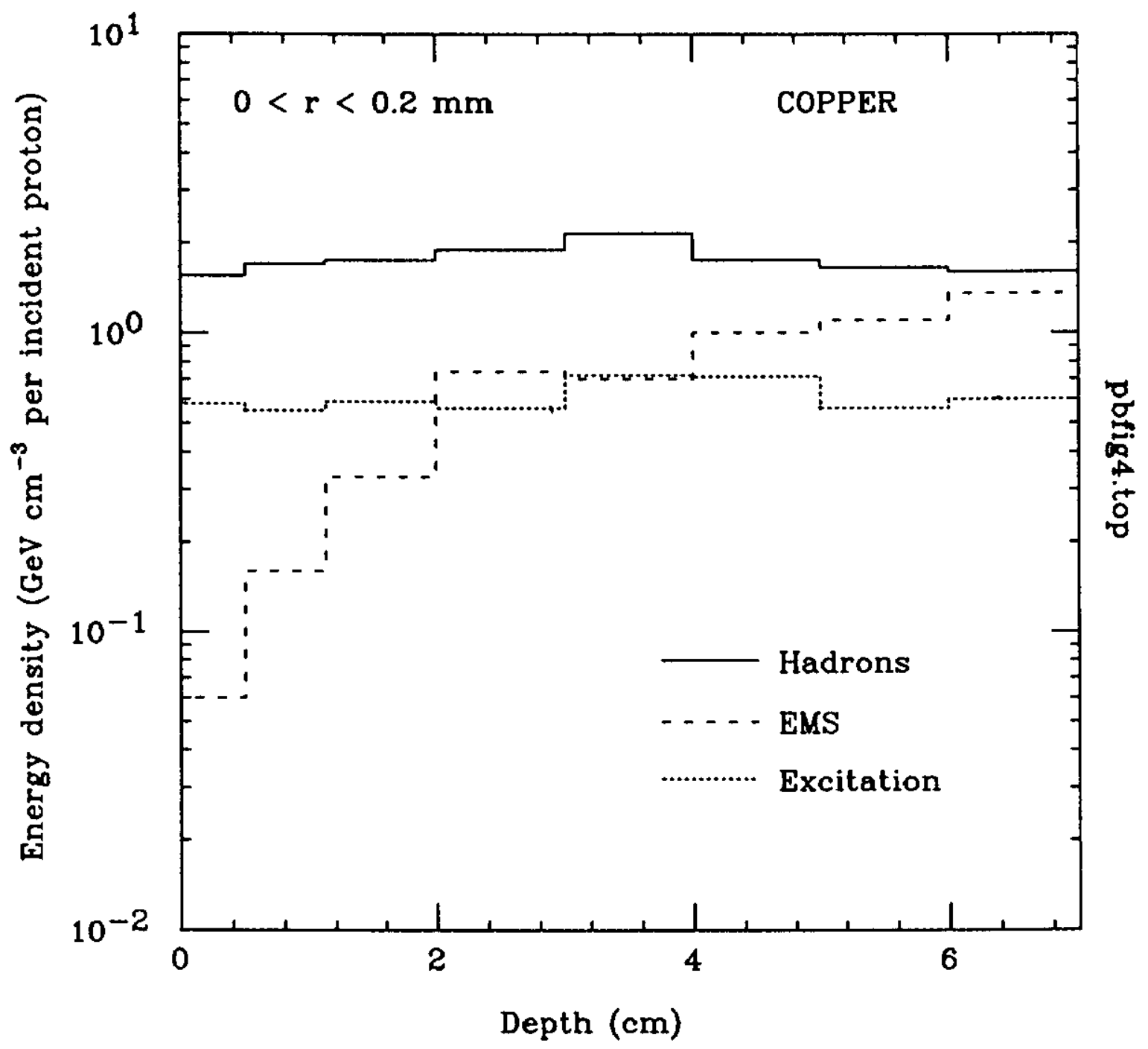

Fig.4. The same, a in fig.3, for copper targot. 


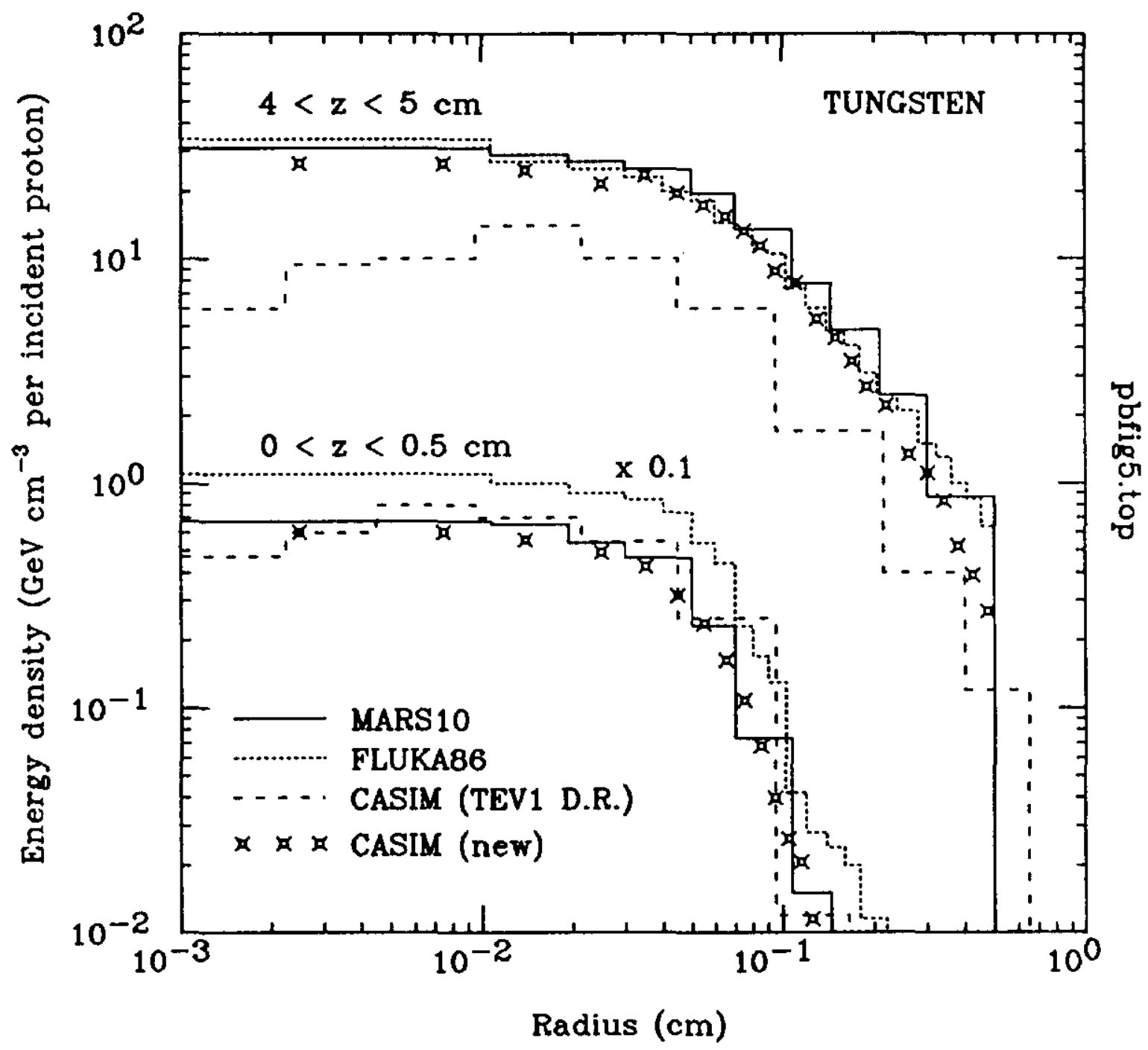

Fig.5. Radiel distibutions of energy density at two depthe of the Pbar tungsten target as calculated with three programs. The upstream results are multiplied by 0.1 . 


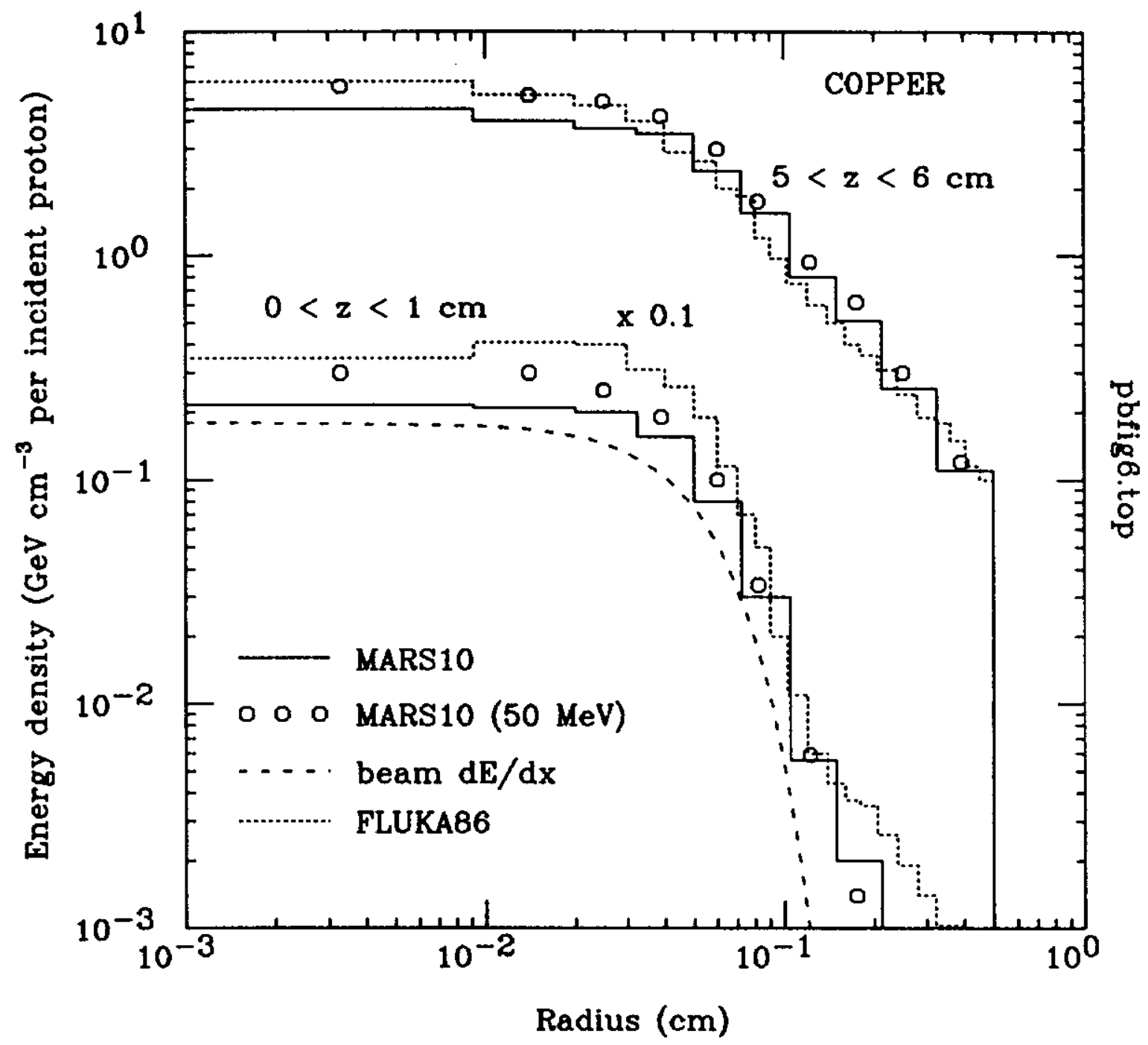

Fig.6. The same, as in fig.5, for copper target colculated with the MARS10 and FLUKABB programs. For the small depth 1 lso shown are the MARS10 date obtained with the increased as in FLUKABB threshold energies and ionization losses of primary protons. 


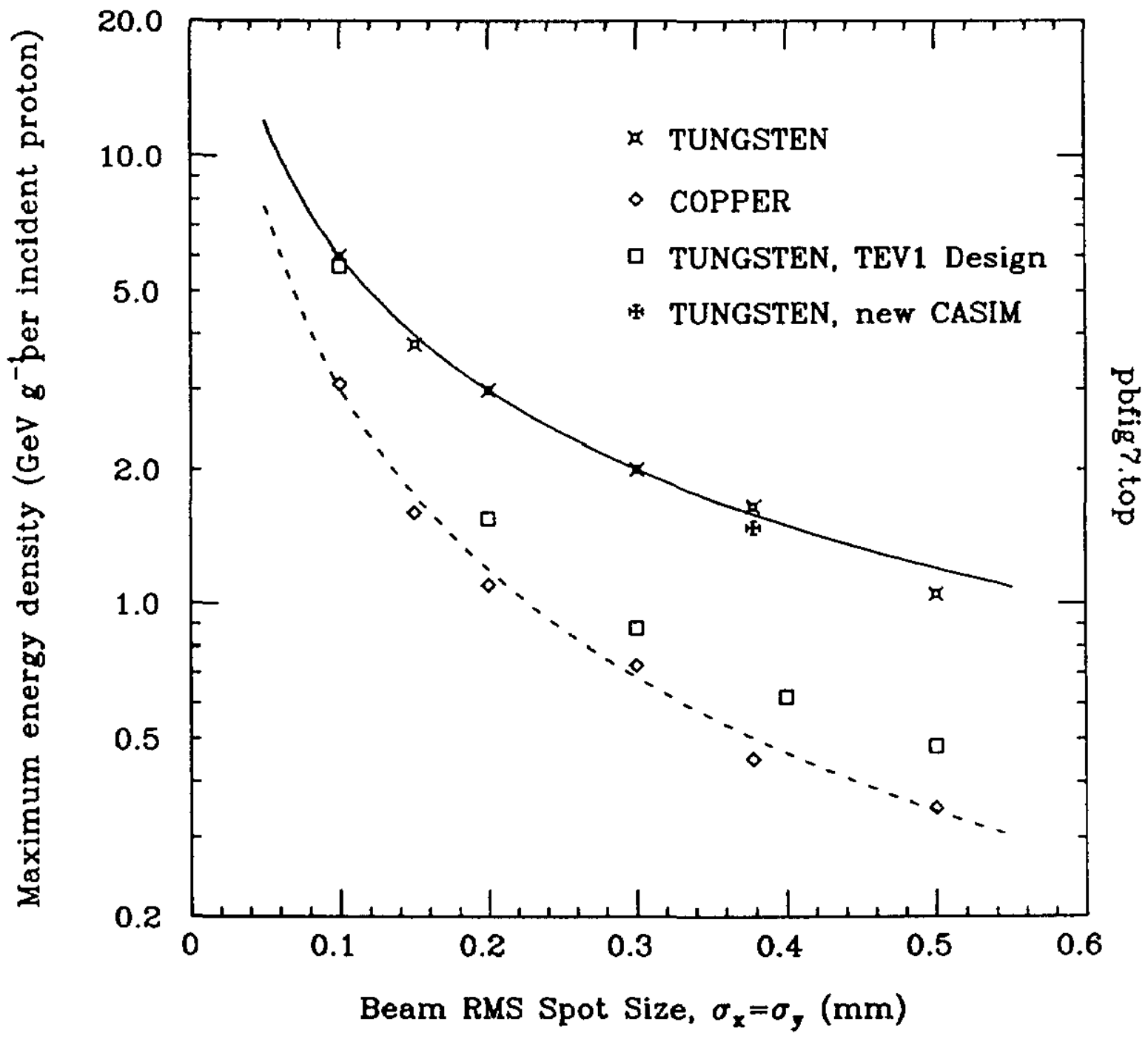

Fig.7. Dependence on the proton beam size of the maximum energy density in the Pbar tungsten and copper targets as calculated with MARS10. Curves are the fits described in the text. Also shown for tungsten ore the Design Report data [4] and the new CASIM result. 


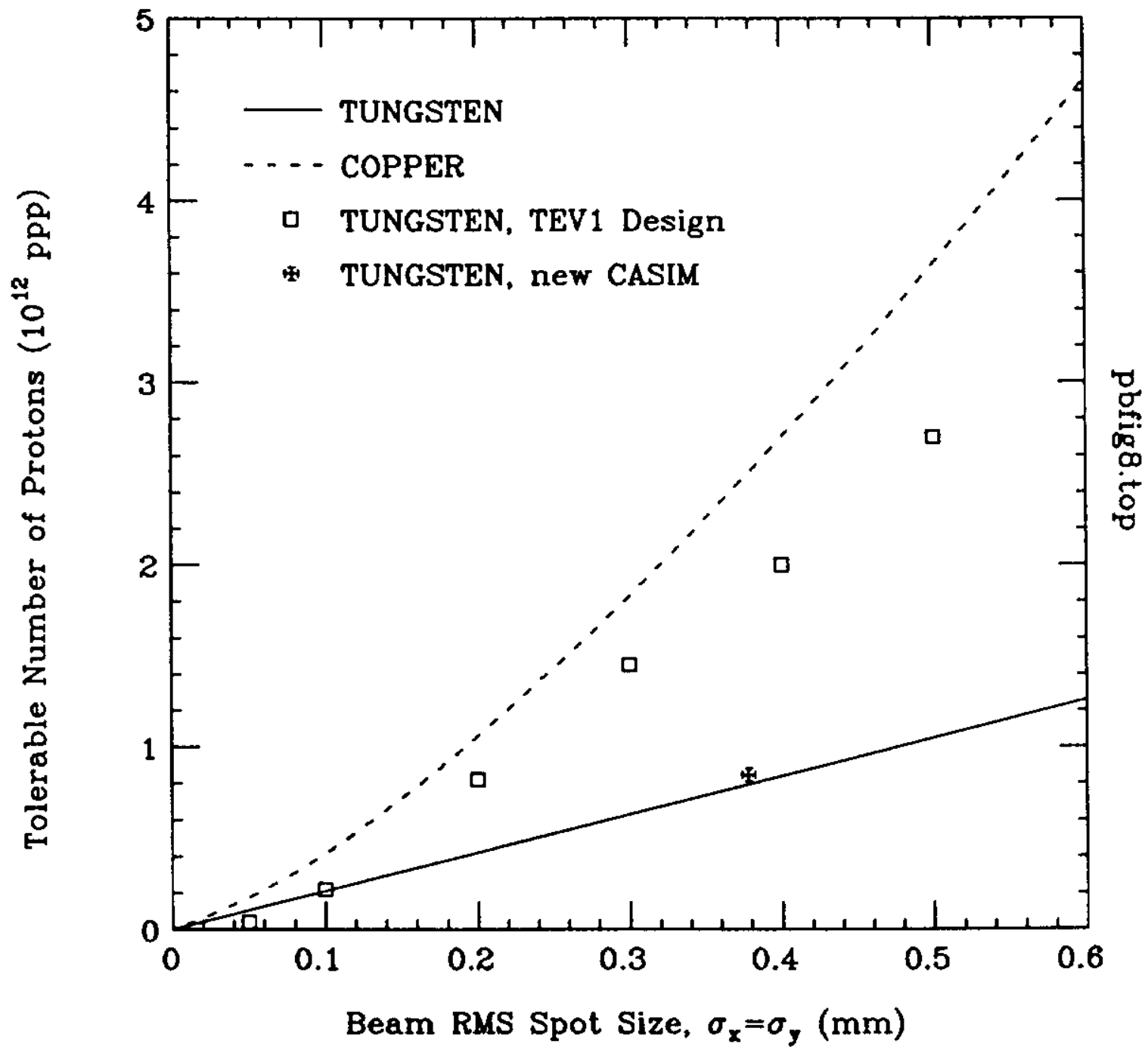

Fig.8. Tolerable number of protons on the Pbar tungsten and copper targets at $120 \mathrm{GoV} / \mathrm{c}$ as function of the beam spot size. Also shown are tungsten data from rof. [4] and the now CASIM result. 